\title{
mTOR-activating mutations in RRAGD cause \\ kidney tubulopathy and cardiomyopathy (KICA) syndrome
}

Karl P. Schlingmann ${ }^{1 *}$, François Jouret ${ }^{2,3 *}$, Kuang Shen ${ }^{4,5,6,7,8 *}$, Anukrati Nigam ${ }^{9}$,

Francisco J. Arjona ${ }^{10}$, Claudia Dafinger ${ }^{11,12}$, Pascal Houillier ${ }^{13,14,15}$, Deborah P. Jones ${ }^{16}$, Felix Kleinerüschkamp ${ }^{17}$, Jun $\mathrm{Oh}^{18}$, Nathalie Godefroid ${ }^{19}$, Mehmet Eltan ${ }^{20}$, Tülay Güran ${ }^{20}$, Stéphane Burtey ${ }^{21}$, Marie-Christine Parotte ${ }^{22}$, Jens König ${ }^{1}$, Alina Braun ${ }^{11,12}$, Caro Bos ${ }^{10}$, Maria Ibars Serra ${ }^{10}$, Holger Rehmann ${ }^{23}$, Fried J.T. Zwartkruis ${ }^{23}$, Kirsten Y. Renkema ${ }^{9}$, Karin Klingel ${ }^{24}$, Eric Schulze-Bahr ${ }^{25}$, Bernhard Schermer ${ }^{12,26}$, Carsten Bergmann ${ }^{27,28}$, Janine Altmüller ${ }^{29}$, Holger Thiele ${ }^{29}$, Bodo B. Beck ${ }^{29}$, Karin Dahan ${ }^{30,31}$, David Sabatini $^{4,5,6,7}$, Max C. Liebau ${ }^{11,12}$, Rosa Vargas-Poussou ${ }^{32}$, Nine V.A.M. Knoers ${ }^{33}$, Martin Konrad $^{1 *}$, Jeroen H.F. de Baaij ${ }^{10 *}$

* These authors contributed equally to this work

Running Title: RRAGD mutations cause KICA syndrome

Word Count: 1990 words

Keywords: mTOR, Rag Complex, Hypomagnesemia, Nephrocalcinosis

\section{Corresponding authors:}

Karl P. Schlingmann

Department of General Pediatrics, University Children`s Hospital Münster Albert Schweitzer Campus 1, 48149 Münster, Germany

Phone: +49-251 835 6214, Email: karlpeter.schlingmann@ukmuenster.de

Jeroen H.F. de Baaij

Department of Physiology, Radboud institute for molecular life sciences, Radboudumc P.O. Box 9101, 6500HB, Nijmegen, The Netherlands

Phone: +31- 24361 7347, Email: jeroen.debaaij@radboudumc.nl 


\section{Affiliations}

${ }^{1}$ Department of General Pediatrics, University Children's Hospital, Münster, Germany

${ }^{2}$ Division of Nephrology, Department of Internal Medicine, University of Liège Hospital, Liège, Belgium

${ }^{3}$ Groupe Interdisciplinaire de Géno-protéomique Appliquée, Cardiovascular Sciences, University of Liège, Liège, Belgium

${ }^{4}$ Whitehead Institute for Biomedical Research, Cambridge, MA, USA

${ }^{5}$ Department of Biology, Howard Hughes Medical Institute, Massachusetts Institute of Technology, Cambridge, MA, USA

${ }^{6}$ Koch Institute for Integrative Cancer Research, Cambridge, MA, USA

${ }^{7}$ Broad Institute of Harvard and Massachusetts Institute of Technology, Cambridge, MA, USA

${ }^{8}$ Program in Molecular Medicine, University of Massachusetts Medical School, Worcester, MA, USA

${ }^{9}$ Department of Genetics, Center for Molecular Medicine, University Medical Center Utrecht, Utrecht University, Utrecht, The Netherlands

${ }^{10}$ Department of Physiology, Radboud Institute for Molecular Life Sciences, Radboud university medical center, Nijmegen, The Netherlands.

${ }^{11}$ Department of Pediatrics and Center for Molecular Medicine Cologne, University of Cologne, Faculty of Medicine and University Hospital Cologne, Cologne, Germany

${ }^{12}$ Department II of Internal Medicine and Center for Molecular Medicine Cologne, University of Cologne, Faculty of Medicine and University Hospital Cologne, Cologne, Germany

${ }^{13}$ Centre de Recherche des Cordeliers, CNRS, ERL8228, INSERM, Sorbonne Université, Université de Paris, F-75006 Paris, France

${ }^{14}$ Department of Physiology, Assistance Publique-Hôpitaux de Paris, Hôpital Européen Georges Pompidou, Paris, France.

${ }^{15}$ Centre de Référence des Maladies Rénales Héréditaires de l'Enfant et de l'Adulte (MARHEA), F75015, Paris, France.

${ }^{16}$ Department of Pediatrics, Vanderbilt University Medical Center, Nashville, TN, USA

17 Department of Pediatric Cardiology, University Children's Hospital, Münster, Germany

${ }^{18}$ Department of Pediatrics, University Medical Center Hamburg-Eppendorf, Hamburg, Germany.

${ }^{19}$ Division of Pediatric Nephrology, Cliniques Universitaires Saint-Luc, UCLouvain, Brussels, Belgium

${ }^{20}$ Marmara University School of Medicine. Department of Pediatric Endocrinology and Diabetes 
${ }^{21}$ Centre de Néphrologie et Transplantation Rénale, AP Hôpitaux de Marseille, Université Aix-

Marseille, Marseille, France

${ }^{22}$ Division of Nephrology-Dialysis, Department of Internal Medicine, CHR Verviers East Belgium, Verviers, Belgium

${ }^{23}$ Department of Molecular Cancer Research, Center for Molecular Medicine, Oncode Institute, University Medical Center Utrecht, Utrecht, The Netherlands

${ }^{24}$ Cardiopathology, Institute for Pathology and Neuropathology, University Hospital Tübingen,

Tübingen, Germany

25 Institute for Genetics of Heart Diseases (IfGH), Department of Cardiovascular Medicine, University Hospital Münster, Münster, Germany

${ }^{26}$ CECAD, University of Cologne, Faculty of Medicine and University Hospital Cologne, Cologne, Germany

${ }^{27}$ Limbach Genetics, Medizinische Genetik Mainz, Prof. Bergmann \& Kollegen, Mainz, Germany

${ }^{28}$ Department of Medicine, Division of Nephrology, University Hospital Freiburg, Germany

${ }^{29}$ Cologne Center for Genomics, University of Cologne, Cologne, Germany

${ }^{30}$ Center of Human Genetics, Gosselies, Belgium

${ }^{31}$ Division of Nephrology, Cliniques Universitaires Saint-Luc, UCLouvain, Brussels, Belgium

${ }^{32}$ Department of Genetics, Assistance Publique Hôpitaux de Paris, Hôpital Européen Georges-

Pompidou, Paris, France

${ }^{33}$ Department of Genetics, University Medical Center Groningen, University of Groningen, Groningen, The Netherlands 


\section{ABSTRACT}

Background - Over the last decaces, advances in genetic techniques have resulted in the identification of rare hereditary disorders of renal magnesium and salt handling. Nevertheless, $\pm 20 \%$ of all tubulopathy patients remain without genetic diagnosis. Here, we explore a large multicentric patient cohort with a novel inherited salt-losing tubulopathy, hypomagnesemia and dilated cardiomyopathy (DCM).

Methods - Whole exome and genome sequencings were performed with various subsequent functional analyses of identified $R R A G D$ variants in vitro.

Results - In 8 children from unrelated families with a tubulopathy characterized by hypomagnesemia, hypokalemia, salt-wasting, and nephrocalcinosis, we identified heterozygous missense variants in RRAGD that mostly occurred de novo. Six of these patients additionally suffered from DCM requiring heart transplantation in 3 of them. An additional dominant variant in RRAGD was simultaneously identified in eight members of a large family with a similar renal phenotype. RRAGD encodes GTPase RagD mediating amino acid signaling to the mechanistic target of rapamycin complex 1 (mTORC1). RagD expression along the mammalian nephron include the thick ascending limb and the distal convoluted tubule. The identified $R R A G D$ variants were shown to induce a constitutive activation of mTOR signaling in vitro,

Conclusions - Our findings establish a novel disease phenotype combining kidney tubulopathy and cardiomyopathy (KICA) caused by an activation of mTOR signaling suggesting a critical role of Rag GTPase D for renal electrolyte handling and cardiac function. 


\section{SIGNIFICANCE STATEMENT}

Here, we report on heterozygous variants in RRAGD in patients with profound hypomagnesemia, renal salt wasting, nephrocalcinosis, and dilated cardiomyopathy. The identified RagD variants induce a constitutive activation of mTOR signaling in vitro. These findings not only establish a novel monogenic disorder of the kidney tubule, but demonstrate the essential role of mTOR signaling for distal tubular electrolyte handling and cardiac function. 


\section{INTRODUCTION}

The kidney tubule balances fluid, electrolyte and mineral homeostasis. The genetic deciphering of hereditary disturbances of electrolyte reabsorption by the tubule, collectively known as tubulopathies, has greatly improved the understanding of electrolyte transport along the nephron. $(1,2)$ Over the last decade, we and others have identified critical components of epithelial $\mathrm{Mg}^{2+}$ reabsorption pathways in the thick ascending limb of Henle's loop (TAL) and the distal convoluted tubule (DCT).(2, 3) In the TAL, $50-70 \%$ of the filtered $\mathrm{Mg}^{2+}$ is reabsorbed paracellularly via the claudin16-19 complex.(4) This paracellular $\mathrm{Mg}^{2+}$ transport is dependent on the transcellular salt reabsorption and negatively regulated by basolateral calcium-sensing receptor. $(3,5)$ In the $\mathrm{DCT}, \mathrm{Mg}^{2+}$ reabsorption is mediated by transient receptor potential melastatin type 6 and 7 (TRPM6/TRPM7) channels.(6-12)

In our cohort of patients with hypomagnesemia, we noted a subset of patients sharing a tubulopathy characterized by (i) renal salt wasting, (ii) profound hypomagnesemia, and (iii) nephrocalcinosis, combined with a dilated cardiomypathy (DCM). As DCM had not been described in patients with known hereditary forms of hypomagnesemia,(2) we hypothesized that these individuals were affected by a yet undefined disease phenotype. By next-generation sequencing, we identified $R R A G D$ variants in eight unrelated individuals, as well as in eight members of a large family with symptomatic hypomagnesemia. 


\section{METHODS}

\section{Subjects}

We initially studied a cohort of 6 individuals with a phenotype combining DCM and a complex renal tubular disorder, including hypomagnesemia (median serum magnesium level of 0.42 $\mathrm{mmol} / \mathrm{L})$. Our second cohort consisted of 3 additional families in which the index cases presented with hypomagnesemia, hypokalemia, hypercalciuria, and nephrocalcinosis. Clinical and biological data at the time of disease manifestation were collected retrospectively from medical charts. We reevaluated all patients and obtained follow-up biochemical data. The detailed clinical history of each individual is provided as Supplemental Materials. All genetic studies were approved by the local ethics committees in Paris, Münster and Liège, and informed consent was obtained from all subjects and/or their parents.

Serum magnesium, potassium, and chloride levels of individuals with RRAGD mutations were statistically compared to non-affected family members. Samples were tested for normal distribution using the Kolmogorov-Smirnov test. For statistical analyses, a one-sided Student t-test in case of normal distribution and a one-sided Mann-Whitney $U$ test in case of nonnormal distribution were used.

\section{Sequencing}

Patients F1.1 to F4.1 were subjected to WES (for details see Supplementary Appendix). Exome data were analyzed for shared genes with variants under all modes of inheritance. After discovery of heterozygous $R R A G D$ variants in these 4 patients, 2 additional patients with DCM and renal tubulopathy were analyzed by conventional Sanger sequencing (F5.1, F6.1). Then, the analyses were expanded to 25 patients with a similar renal tubular disorder, but without known cardiac phenotype that revealed 2 additional patients (F7.1, F8.1).(40) In short, genomic DNA was fragmented, amplified and sequenced simultaneously by Illumina next generation sequencing technology using an Illumina NextSeq system. DNA samples of members III.1, III.6, and IV.2 family 9 were subjected to WGS on the Illumina X Ten platform (Hartwig Medical Foundation Amsterdam, The Netherlands). Data analysis was performed by 
mapping paired end reads $(2 \times 100 \mathrm{bp})$ from the NextSeq instrument against the hg19 human reference genome using Burrows Wheeler Aligner with recommended standard settings. Filtering steps excluded common SNPs (> 1\%), introns, untranslated regions (UTR), synonymous variants and low-coverage regions $(<5$ reads). Overlaps of the remaining variants were generated using VCFMiner to select genes that were present in F1.1-F4.1, which resulted in the identification of one remaining gene, $R R A G D .(41)$ Subsequently, individuals F5.1 and F6.1 were subjected to conventional Sanger sequencing of the complete RRAGD gene. An extensive description of the sequencing procedure has been provided in the Supplemental Data.

Due to the lack of genetic material, a RRAGD mutation could not be confirmed in the affected father of individual F4.1 who died from DCM at 41 years of age. Of note, an older sister of individual F7.1 died in infancy from acute cardiac failure during a pneumonia episode and the autopsy revealed DCM. Careful genetic reevaluation revealed mosaicism (17\%) in leucocytes of the healthy mother for the respective RRAGD mutation (see Supplemental Data).

\section{Cell Lysates and Immunoprecipitation}

Human embryonic kidney (HEK293T) cells were transfected with Flag-S6K1, RagA and RagD mutants as described previously.(23) To determine the response of mTORC1 to amino acid stimulation or starvation under the control of Rag mutants, two million HEK293T cells were plated onto a 10-cm dish. Twenty-four hours later, the cells were transfected with $2 \mathrm{ng}$ of S6K1 and $100 \mathrm{ng}$ of each Rag wildtype or mutant construct. Thirty-six hours later, amino acid stimulation or starvation was performed for 0.5-1 hour. Cells were rinsed once with ice-cold PBS and lysed with Triton lysis buffer (40 mM Na-HEPES, pH 7.4; 5 mM MgCl2; 100 mM ATP; $10 \mathrm{mM} \mathrm{Na4P2O7;} 10 \mathrm{mM} \mathrm{Na} \beta$-glycerol phosphate; $1 \% \mathrm{v} / \mathrm{v}$ Triton; and one tablet of protease inhibitor cocktail per $25 \mathrm{~mL}$ of buffer). The lysates were cleared by centrifugation at 15,000 rpm at $4^{\circ} \mathrm{C}$ in a microcentrifuge for 10 minutes. For immunoprecipitations, the FLAGM2 beads were pre-equilibrated in Triton lysis buffer. $30 \mu \mathrm{L}$ of a $50 / 50$ slurry of the FLAG-M2 affinity beads were then added to cleared lysates and incubated at $4^{\circ} \mathrm{C}$ for two hours. Following 
immunoprecipitation, the beads were washed one time with Triton lysis buffer and 3 times with Triton lysis buffer supplemented with $500 \mathrm{mM} \mathrm{NaCl}$. Immunoprecipitated proteins were denatured by the addition of $50 \mu \mathrm{L}$ of $2.5 \times$ SDS buffer, resolved by SDS-PAGE, and analyzed by immunoblotting.

\section{Sample preparation for interactome analysis}

FIpIn-IMCD (inner medullary collecting duct) cells stably expressing GFP-GFP, GFP-Rragd-wt or GFP-Rragd-Ser75Leu fusion proteins were generated as described previously.(42) Lysates were cleared by centrifugation, followed by incubation with GFP $\mu$ MACS magnetic beads (Miltenyi) for $1 \mathrm{~h}$. Then, lysates were loaded on a $\mu$ MACS column and precipitated proteins were reduced, alkylated and on-column digestion was performed over-night as previously described. Eluates were acidified the next day using 1-2\% formic acid and stage-tip clean-up was performed as previously described and samples were dried using a vacuum centrifuge. Peptides were resuspended in $0.1 \%$ FA and were separated using a $1 \mathrm{~h}$ gradient on an $\mathrm{nLC}$ coupled to a Q Exactive Plus tandem mass spectrometer (Thermo scientific) or an LTQ orbitrap $\mathrm{XL}$ mass spectrometer. Experimental details are provided in the Supplemental Data.

\section{Expression analysis}

Total RNA was extracted from issues of C57BL/6 mice and isolated distal convoluted tubule (DCT) cells of parvalbumin-GFP mice using Trizol Reagent (Thermo Fisher Scientific, Waltham, MA, USA) according to manufacturer's protocol. To remove genomic DNA, the isolated RNA was subsequently subjected to DNase (Promega, Madison, WI, USA) treatment. RNA was reverse transcribed using Moloney murine leukemia virus reverse transcriptase (Thermo Fisher Scientific) for $1 \mathrm{~h}$ at $37^{\circ} \mathrm{C}$. Ras-related GTP binding D (Rragd) (forward, CACCTGAGCTTTTACCTGA; reverse, TCAGCAGATTCTCCAGCGTC) gene expression levels were quantified by SYBR-Green (Bio-Rad) on a CFX96 Real-Time PCR Detection System (Bio-Rad) and normalized for glyceraldehyde 3-phosphate dehydrogenase (Gapdh) 


\section{(forward TAACATCAAATGGGGTGAGG; reverse, GGTTCACACCCATCACAAAC)} expression levels.

Immunohistochemistry was performed on kidney tissues of C57BL/6 mice using primary antibodies against RagD, AQP2, NCC, and uromodulin as described previously (43). Paraffinebedded kidney sections were subjected to deparaffinization and rehydration, followed by permeabilization in PBS-Triton $0.3 \%(\mathrm{v} / \mathrm{v})$ (Sigma). Sections were incubated overnight at $4^{\circ} \mathrm{C}$ with the primary antibodies sheep anti-NCC (1:400) (Sheep S965B, MRC PPU, Dundee), sheep anti-Tamm Horsfall (1:200) (MBS220487, Bio-Trend) and guinea pig anti-AQP2 (1:100) (kindly provided by dr. Peter Deen) and subsequently incubated overnight at $4^{\circ} \mathrm{C}$ with the primary antibody rabbit anti-RRAGD (1:2,000) (NBP2-32106, Novus Biologicals). Experimental details are provided in the Supplemental Data. 


\section{RESULTS}

\section{Patients}

We initially identified eight unrelated individuals with hypomagnesemia and a complex renal tubulopathy phenotype (Table 1), accompanied by muscle spasms and seizures in three individuals. Inappropriately high fractional excretion rates of magnesium indicated renal magnesium wasting (FE-Mg, Table 1). In individual F2.1, a magnesium loading test was performed, which disclosed a decreased renal tubular threshold for magnesium reabsorption (Figure 1A). Additional renal findings included polyuria, hypokalemia, hypochloremia, a tendency towards metabolic alkalosis, hypercalciuria, and nephrocalcinosis pointing to a defect in the TAL (Table 1, Figure 1B-E). Of note, the prenatal course of individuals F3.1 and F4.1 was complicated by polyhydramnios, as observed in antenatal Bartter syndrome.(13). All family members of these probands were clinically unaffected. In six patients (F1-F6), the tubulopathy was accompanied by DCM, resulting in terminal heart failure and requiring heart transplantation in individuals F1.1 to F3.1 (Figure 1F).

In parallel of these eight unrelated patients, we analyzed a large family (F9) with eight affected members presenting with profound and symptomatic hypomagnesemia resistant to adequate oral magnesium supplements (pedigree and clinical details in Supplementary Figure 1 \& Supplementary Table 1, respectively). The index case presented in early adulthood with muscle cramps and tetany. In addition, affected individuals exhibited hypokalemia, metabolic alkalosis and an activation of the renin-angiotensin-aldosterone system (RAAS) together with normal or low blood pressure suggesting the diagnosis of Gitelman syndrome (Table 1, Figure 1C). None of these patients showed cardiac abnormalities.

Serum potassium and chloride levels were significantly lower in all affected individuals $(n=16)$ compared with those of available non-affected family members $(n=8)$ (Figure 1D-E), which, together with metabolic alkalosis and RAAS activation observed in family 9 , was highly indicative of renal salt wasting. 
After exclusion of mutations in known genes for hypomagnesemia and salt wasting, we performed whole-exome sequencing (WES) in individuals F1.1 to F4.1, which revealed $R R A G D$ as a single common mutated gene. The identified $R R A G D$ variants were all present in heterozygous state and mostly occurred de novo. Subsequently, additional heterozygous RRAGD variants were identified in four sporadic individuals F5.1 to 8.1. Family F9 was analyzed separately under the assumption of a dominant trait. Whole genome sequencing (WGS) revealed a heterozygous $R R A G D$ variant ( $\mathrm{p}$.Thr97Pro) that co-segregated with the disease phenotype.

$R R A G D$ encodes a small Rag guanosine triphosphatase (GTPase), which is a member of the Ras family of GTP-binding proteins.(14) All RagD mutations affect highly conserved amino acid residues in GTP-binding domains conserved in small GTPases (G-domains, G1 to G5, Figure 2A-D, Supplementary Figure 2).(15)

RagD showed a ubiquitous expression pattern with significant transcript levels in heart and kidney (Supplementary Figure 3). Compared to well-established markers of distal tubular segments, RagD co-localized with uromodulin (UMOD) and $\mathrm{Na}^{+}-\mathrm{Cl}^{-}$-cotransporter (NCC) in the TAL and DCT nephron segments, respectively (Figure 2E-G).

\section{RagD mutations activate the mTOR pathway}

All RagD mutants showed increased binding to Raptor (regulatory associated protein of mTOR) and mTOR, the essential components of mTORC1 (Figure $3 \mathrm{~A}$ ) after exogenous expression in HEK293T cells. All human RagD mutants resulted in increased S6K1 phosphorylation in the presence of nutrients, indicative of active mTORC1 signaling. Of note, some mutants increased S6K1 phosphorylation even during starvation (Figure 3B).

To further evaluate the effect of human RagD-p.Ser76Leu on mTORC1 complexes, we performed an interactome analysis by quantitative proteomics after stable genomic integration in a murine tubular epithelial cell line (Figure 3C). Here, mutant RagD-p.Ser75Leu showed a significantly increased co-precipitation of multiple proteins of the mTORC1 signaling complex (Figure 3D), includingRaptor, members of the Lamtor protein family (Lamtor3, Lamtor5) and 
different subunits of lysosomal vacuolar-type $\mathrm{H}^{+}$-ATPase (V-ATPase, Atp6v-proteins), (Figure 3C-D, Supplementary Figure 4).

\section{DISCUSSION}

The present study describes a previously unrecognized hereditary disease phenotype combining an electrolyte-losing tubulopathy and DCM that is caused by heterozygous missense variants in the RRAGD gene encoding Rag GTPase D. The identified RRAGD variants lead to increased mTOR signaling. We propose to call this novel inherited disease: "the KICA (Kidney tubulopathy and Cardiomyopathy) syndrome".

The cardinal symptom of individuals with RRAGD mutations is a severe and substitution-resistant hypomagnesemia, resulting in muscle cramps, tetany, and cerebral seizures. Renal magnesium wasting was associated with hypercalciuria and nephrocalcinosis in a subset of individuals, a phenotype highly reminiscent of FHHNC (Familial Hypomagnesemia with Hypercalciuria and Nephrocalcinosis; OMIM \#248250, \#248190)(2, 3). In FHHNC, mutations in two tight junction proteins, claudin-16 and claudin-19, cause a defective paracellular reabsorption of calcium and magnesium in the TAL.(16-18) Immunostaining experiments indeed showed RagD expression in the TAL, suggesting that RagD variants may impair $\mathrm{Ca}^{2+}$ and $\mathrm{Mg}^{2+}$ reabsorption in this tubular segment.

In a subset of patients, a renal phenotype with salt wasting and hypokalemic alkalosis prevailed resembling Bartter syndrome. The prenatal finding of polyhydramnios in three individuals supported this assumption. Unfortunately, a systematic evaluation of the RAAS in our entire cohort was hampered by early cardiac insufficiency and treatment with diuretics and ACE inhibitors in most individuals. However, a marked activation of the RAAS was also noted in individual F2.1 years before cardiac manifestation supporting the suspicion of a mixed FHHNC/salt wasting phenotype. On the other hand, hypercalciuria and nephrocalcinosis lacked in affected members of family 9 . This phenotypic variability might be explained by a less pronounced activation of mTOR signaling by the RagD-p.T97P mutant, but could also reflect a primary dysfunction of the DCT with a Gitelman syndrome-like phenotype rather than 
a TAL defect. Similar observations have been made in Bartter syndrome type III due to CLCNKB defects, in which individuals might present with a phenotype ranging from antenatal Bartter syndrome to Gitelman syndrome.(19, 20) Future research will be required to elucidate the exact targets of increased mTOR signaling in distal tubular segments.

Interestingly, the phenotype of our cohort is reminiscent of 2 individuals from a previously described Finnish family with unresolved renal tubular disease and cardiomyopathy. $(21,22)$ The clinical picture of these individuals is highly suggestive of KICA syndrome. Unfortunately, the cardiac phenotype resulted in an early fatal issue in this family, which hampers any further genetic investigation.

RRAGD encodes a small Rag guanosine triphosphatase (GTPase), which is an essential component of the nutrient-sensing pathway that activates mTOR signaling (14). mTOR serves as the main nutrient sensor of the cell coordinating signals from extracellular growth factors and intracellular nutrient availability, such as amino acids.(23) (24) Upon amino acid stimulation, Rag GTPases target the mTOR complex1 (mTORC1) to the lysosome where its kinase is activated.(23) Indeed, our interaction studies demonstrate increased interaction of RagD-S76L to components of the mTOR signaling pathway and the Ragulator complex (Figure 3). The human RRAGD mutations discovered in the present study affect residues involved in the binding of guanosine nucleotides (Figure 2, Supplementary Figure 2). Previously, engineered mutations that interfere with nucleotide binding have been shown to critically affect Rag signaling and mTORC1 activation. $(23,25)$ In line with this, the variants discovered here lead to an increased activation of mTORC1, a subset even under amino acid starvation (Figure 3).

Data on the role of mTOR signaling in tubular physiology is still scarce. Previous studies mainly focused on the tubular effects of decreased mTORC1 signaling. $(26,27)$ Inactivation of mTOR signaling along the renal tubule was shown to lead to a Fanconi-like syndrome as well as a urinary concentrating defect attributed to impaired distal tubular transport processes.(28) 
Remarkably, hypomagnesemia and hypokalemia have been observed in rats after sirolimus treatment that were attributed to reduced expression of NKCC2 (SLC12A1) in the TAL.(29) Moreover, inhibition of mTOR signaling also resulted in changes in TRPM6 expression in the DCT.(30) Conversely, mTORC1 activation by knock-out of Tsc1 in renal tubular cells resulted in cyst formation resembling polycystic kidney disease (PKD).(31, 32) Our findings now underline the indispensable role of $\mathrm{mTORC} 1$ signaling for tubular electrolyte handling that appears to be particularly sensitive to both inactivation and hyperactivity of the mTOR pathway.

The finding of DCM in a subset of our patients also substantiates a critical role of Rag GTPases for cardiac function. Previously, inactivation of Rag GTPases RagA and RagB was shown to result in hypertrophic cardiomyopathy in mice.(33) Moreover, a de novo mutation in RagC (p.Ser75Tyr) leading to increased mTOR activity has been described in a single individual with DCM.(34) Recent studies in clinical and experimental models of DCM have implicated mTOR hyperactivation as a major cause of DCM. $(35,36)$ In line, inhibition of mTOR by rapamycin has been demonstrated to successfully improve cardiac function in animal models of DCM.(37-39) Of note, not all identified RRAGD mutations overactivated mTORC1 signaling equally. Our experiments indicate that the p.Thr97Pro results in less S6K1 activation (Figure 3), which may explain why individuals carrying this mutation do not suffer from DCM even at adult age.

Taken together, the discovery of RRAGD mutations establishes a novel hereditary tubulopathy linked to dysregulation of the mTOR pathway. The diagnosis of KICA syndrome should be considered in individuals presenting either with early-onset DCM and/or hypomagnesemia of renal origin. Future research will hopefully help to enlighten the exact role of mTOR for distal tubular transport processes and renal magnesium handling in particular. 


\section{DISCLOSURES}

M.L. has received honoraria for scientific lectures from Pfizer. Representing the University Hospital of Cologne, M.L. has been counselling Otsuka Pharmaceuticals in an advisory board. The other authors have no conflict of interest to disclose.

\section{ACKNOWLEDGEMENTS}

The authors are grateful to the patients for their participation in this study. We thank Edith Peters, Femke Latta, Valentina Carotti, Ruud Tilleman, Matthijs Snelders, Marie Neuville, and

Ruth Herzog for their excellent technical support and Tanja Seidel for thoughtful patient care. This work was supported by grants from the Netherlands Organization for Scientific Research (NWO VENI 016.186.012), the Dutch Diabetes Research Foundation (2017.81.014), the Dutch Kidney Foundation (15OKG18 to KYR), and the EURenOmics project from the European Union seventh Framework Program (FP7/2007-2013, no. 305608). FJ is a Fellow of the Fonds National de la Recherche Scientifique (FNRS) in Belgium. 


\section{SUPPLEMENTAL DATA - TABLE OF CONTENTS}

\section{Text}

1. Complete methods

2. Detailed clinical description of affected individuals

3. Detailed description of the structural consequences of the mutations

4. References

\section{Tables}

1. Biochemical parameters F9

\section{Figures}

\section{Pedigree F9}

2. RagD mutations affect conserved residues

3. Tissue expression of RagD

4. Effects RagD mutations on its interactome 


\section{REFERENCES}

1. Downie ML, Lopez Garcia SC, Kleta R, Bockenhauer D: Inherited Tubulopathies of the Kidney: Insights from Genetics. Clin J Am Soc Nephrol, 2020 10.2215/CJN.14481119

2. Viering D, de Baaij JHF, Walsh SB, Kleta R, Bockenhauer D: Genetic causes of hypomagnesemia, a clinical overview. Pediatr Nephrol, 32: 1123-1135, 2017 $10.1007 / \mathrm{s} 00467-016-3416-3$

3. de Baaij JH, Hoenderop JG, Bindels RJ: Magnesium in man: implications for health and disease. Physiol Rev, 95: 1-46, 2015 10.1152/physrev.00012.2014

4. Hou J, Renigunta A, Gomes AS, Hou M, Paul DL, Waldegger S, et al.: Claudin-16 and claudin-19 interaction is required for their assembly into tight junctions and for renal reabsorption of magnesium. Proc Natl Acad Sci U S A, 106: 15350-15355, 2009 10.1073/pnas.0907724106

5. Gong Y, Renigunta V, Himmerkus N, Zhang J, Renigunta A, Bleich M, et al.: Claudin-14 regulates renal $\mathrm{Ca}(+)(+)$ transport in response to CaSR signalling via a novel microRNA pathway. EMBO J, 31: 1999-2012, 2012 10.1038/emboj.2012.49

6. Mittermeier L, Demirkhanyan L, Stadlbauer B, Breit A, Recordati C, Hilgendorff A, et al.: TRPM7 is the central gatekeeper of intestinal mineral absorption essential for postnatal survival. Proc Natl Acad Sci U S A, 116: 4706-4715, 2019 10.1073/pnas.1810633116

7. Schaffers OJM, Hoenderop JGJ, Bindels RJM, de Baaij JHF: The rise and fall of novel renal magnesium transporters. Am J Physiol Renal Physiol, 314: F1027-F1033, 2018 10.1152/ajprenal.00634.2017

8. Schlingmann KP, Weber S, Peters M, Niemann Nejsum L, Vitzthum H, Klingel K, et al.: Hypomagnesemia with secondary hypocalcemia is caused by mutations in TRPM6, a new member of the TRPM gene family. Nat Genet, 31: 166-170, $200210.1038 / n g 889$

9. de Baaij JH, Blanchard MG, Lavrijsen M, Leipziger J, Bindels RJ, Hoenderop JG: P2X4 receptor regulation of transient receptor potential melastatin type 6 (TRPM6) Mg2+ channels. Pflugers Arch, 466: 1941-1952, 2014 10.1007/s00424-014-1440-3 
10. Ferioli S, Zierler S, Zaisserer J, Schredelseker J, Gudermann T, Chubanov V: TRPM6 and TRPM7 differentially contribute to the relief of heteromeric TRPM6/7 channels from inhibition by cytosolic Mg(2+) and Mg.ATP. Sci Rep, 7: 8806, 2017 10.1038/s41598017-08144-1

11. Nair AV, Hocher B, Verkaart S, van Zeeland F, Pfab T, Slowinski T, et al.: Loss of insulininduced activation of TRPM6 magnesium channels results in impaired glucose tolerance during pregnancy. Proc Natl Acad Sci U S A, 109: 11324-11329, 2012 10.1073/pnas.1113811109

12. Thebault S, Alexander RT, Tiel Groenestege WM, Hoenderop JG, Bindels RJ: EGF increases TRPM6 activity and surface expression. J Am Soc Nephrol, 20: 78-85, 2009 10.1681/ASN.2008030327

13. Walsh PR, Tse Y, Ashton E, lancu D, Jenkins L, Bienias M, et al.: Clinical and diagnostic features of Bartter and Gitelman syndromes. Clin Kidney J, 11: 302-309, 2018 $10.1093 / \mathrm{ckj} / \mathrm{sfx} 118$

14. Saxton RA, Sabatini DM: mTOR Signaling in Growth, Metabolism, and Disease. Cell, 168: 960-976, 2017 10.1016/j.cell.2017.02.004

15. Nicastro R, Sardu A, Panchaud N, De Virgilio C: The Architecture of the Rag GTPase Signaling Network. Biomolecules, 7, 2017 10.3390/biom7030048

16. Konrad M, Schaller A, Seelow D, Pandey AV, Waldegger S, Lesslauer A, et al.: Mutations in the tight-junction gene claudin 19 (CLDN19) are associated with renal magnesium wasting, renal failure, and severe ocular involvement. Am J Hum Genet, 79: 949-957, $200610.1086 / 508617$

17. Simon DB, Lu Y, Choate KA, Velazquez H, Al-Sabban E, Praga M, et al.: Paracellin-1, a renal tight junction protein required for paracellular Mg2+ resorption. Science, 285: 103-106, 1999 10.1126/science.285.5424.103

18. Hanssen O, Castermans E, Bovy C, Weekers L, Erpicum P, Dubois B, et al.: Two novel mutations of the CLDN16 gene cause familial hypomagnesaemia with hypercalciuria and nephrocalcinosis. Clin Kidney J, 7: 282-285, 2014 10.1093/ckj/sfu019 
19. Jeck N, Konrad M, Peters M, Weber S, Bonzel KE, Seyberth HW: Mutations in the chloride channel gene, CLCNKB, leading to a mixed Bartter-Gitelman phenotype. Pediatr Res, 48: 754-758, 2000 10.1203/00006450-200012000-00009

20. Zelikovic I, Szargel R, Hawash A, Labay V, Hatib I, Cohen N, et al.: A novel mutation in the chloride channel gene, CLCNKB, as a cause of Gitelman and Bartter syndromes. Kidney Int, 63: 24-32, 2003 10.1046/j.1523-1755.2003.00730.x

21. Laine J, Jalanko H, Alakulppi N, Holmberg C: A new tubular disorder with hypokalaemic metabolic alkalosis, severe hypermagnesuric hypomagnesaemia, hypercalciuria and cardiomyopathy. Nephrol Dial Transplant, 20: 1241-1245, 2005 10.1093/ndt/gfh760

22. Runeberg L, Collan Y, Jokinen EJ, Lahdevirta J, Aro A: Hypomagnesemia due to renal disease of unknown etiology. Am J Med, 59: 873-881, 1975 10.1016/00029343(75)90481-7

23. Sancak Y, Peterson TR, Shaul YD, Lindquist RA, Thoreen CC, Bar-Peled L, et al.: The Rag GTPases bind raptor and mediate amino acid signaling to mTORC1. Science, 320: 1496-1501, 2008 10.1126/science.1157535

24. Shimobayashi M, Hall MN: Making new contacts: the mTOR network in metabolism and signalling crosstalk. Nat Rev Mol Cell Biol, 15: 155-162, 2014 10.1038/nrm3757

25. Shen K, Choe A, Sabatini DM: Intersubunit Crosstalk in the Rag GTPase Heterodimer Enables mTORC1 to Respond Rapidly to Amino Acid Availability. Mol Cell, 68: 821, 2017 10.1016/j.molcel.2017.10.031

26. Grahammer F, Haenisch N, Steinhardt F, Sandner L, Roerden M, Arnold F, et al.: mTORC1 maintains renal tubular homeostasis and is essential in response to ischemic stress. Proc Natl Acad Sci U S A, 111: E2817-2826, 2014 10.1073/pnas.1402352111

27. Grahammer F, Wanner N, Huber TB: mTOR controls kidney epithelia in health and disease. Nephrol Dial Transplant, 29 Suppl 1: i9-i18, 2014 10.1093/ndt/gft491

28. Grahammer F, Ramakrishnan SK, Rinschen MM, Larionov AA, Syed M, Khatib H, et al.: mTOR Regulates Endocytosis and Nutrient Transport in Proximal Tubular Cells. J Am Soc Nephrol, 28: 230-241, 2017 10.1681/ASN.2015111224 
29. da Silva CA, de Braganca AC, Shimizu MH, Sanches TR, Fortes MA, Giorgi RR, et al.: Rosiglitazone prevents sirolimus-induced hypomagnesemia, hypokalemia, and downregulation of NKCC2 protein expression. Am J Physiol Renal Physiol, 297: F916922, 2009 10.1152/ajprenal.90256.2008

30. Ikari A, Sanada A, Sawada H, Okude C, Tonegawa C, Sugatani J: Decrease in transient receptor potential melastatin 6 mRNA stability caused by rapamycin in renal tubular epithelial cells. Biochim Biophys Acta, 1808: 1502-1508, 2011 10.1016/j.bbamem.2010.11.006

31. Centini R, Tsang M, Iwata T, Park H, Delrow J, Margineantu D, et al.: Loss of Fnip1 alters kidney developmental transcriptional program and synergizes with TSC1 loss to promote mTORC1 activation and renal cyst formation. PLoS One, 13: e0197973, 2018 10.1371/journal.pone. 0197973

32. Zhou J, Brugarolas J, Parada LF: Loss of Tsc1, but not Pten, in renal tubular cells causes polycystic kidney disease by activating mTORC1. Hum Mol Genet, 18: 4428-4441, 2009 10.1093/hmg/ddp398

33. Kim YC, Park HW, Sciarretta S, Mo JS, Jewell JL, Russell RC, et al.: Rag GTPases are cardioprotective by regulating lysosomal function. Nat Commun, 5: 4241, 2014 $10.1038 /$ ncomms5241

34. Long PA, Zimmermann MT, Kim M, Evans JM, Xu X, Olson TM: De novo RRAGC mutation activates mTORC1 signaling in syndromic fetal dilated cardiomyopathy. Hum Genet, 135: 909-917, 2016 10.1007/s00439-016-1685-3

35. Caragnano A, Aleksova A, Bulfoni M, Cervellin C, Rolle IG, Veneziano C, et al.: Autophagy and Inflammasome Activation in Dilated Cardiomyopathy. J Clin Med, 8, 2019 10.3390/jcm8101519

36. Jin B, Shi H, Zhu J, Wu B, Geshang Q: Up-regulating autophagy by targeting the mTOR4EBP1 pathway: a possible mechanism for improving cardiac function in mice with experimental dilated cardiomyopathy. BMC Cardiovasc Disord, 20: 56, 2020 $10.1186 / \mathrm{s} 12872-020-01365-9$ 
37. Choi JC, Worman HJ: Reactivation of autophagy ameliorates LMNA cardiomyopathy. Autophagy, 9: 110-111, 2013 10.4161/auto.22403

38. Marin TM, Keith K, Davies B, Conner DA, Guha P, Kalaitzidis D, et al.: Rapamycin reverses hypertrophic cardiomyopathy in a mouse model of LEOPARD syndrome-associated PTPN11 mutation. J Clin Invest, 121: 1026-1043, 2011 10.1172/JCI44972

39. Ramos FJ, Chen SC, Garelick MG, Dai DF, Liao CY, Schreiber KH, et al.: Rapamycin reverses elevated mTORC1 signaling in lamin A/C-deficient mice, rescues cardiac and skeletal muscle function, and extends survival. Sci Transl Med, 4: 144ra103, 2012 10.1126/scitransImed.3003802

40. Altmuller J, Motameny S, Becker C, Thiele H, Chatterjee S, Wollnik B, et al.: A systematic comparison of two new releases of exome sequencing products: the aim of use determines the choice of product. Biol Chem, 397: 791-801, 2016 10.1515/hsz-20150300

41. Hart SN, Duffy P, Quest DJ, Hossain A, Meiners MA, Kocher JP: VCF-Miner: GUI-based application for mining variants and annotations stored in VCF files. Brief Bioinform, 17: 346-351, 2016 10.1093/bib/bbv051

42. Dafinger C, Rinschen MM, Borgal L, Ehrenberg C, Basten SG, Franke M, et al.: Targeted deletion of the AAA-ATPase Ruvbl1 in mice disrupts ciliary integrity and causes renal disease and hydrocephalus. Exp Mol Med, 50: 75, 2018 10.1038/s12276-018-0108-z

43. Kurstjens S, Smeets B, Overmars-Bos C, Dijkman HB, den Braanker DJW, de Bel T, et al.: Renal phospholipidosis and impaired magnesium handling in high-fat-diet-fed mice. FASEB J, 33: 7192-7201, 2019 10.1096/fj.201801778RR

44. Hoyer PF: Nephrocalcinose. In: Ultraschalldiagnostik in Pädiatrie und Kinderchirurgie. Stuttgart, Thieme, 1996, pp 372-374

45. Pettersen MD, Du W, Skeens ME, Humes RA: Regression equations for calculation of $z$ scores of cardiac structures in a large cohort of healthy infants, children, and adolescents: an echocardiographic study. J Am Soc Echocardiogr, 21: 922-934, 2008 10.1016/j.echo.2008.02.006 


\section{FIGURE LEGENDS}

Figure 1. Clinical and genetic findings in 9 families with cardiomyopathy and renal tubulopathy

(A) Magnesium loading test in individual F2.1. Urinary magnesium excretion is plotted against the ultrafiltrable fraction of serum magnesium before and during parenteral infusion of magnesium at increasing concentrations (two separate tests). The dashed lines represent the normal range. (B) Renal ultrasound of individual F7.1 showing severe medullary nephrocalcinosis (Grade III according to Hoyer)(44). (C-E) Comparison of serum magnesium, potassium and chloride levels of individuals with RRAGD mutations (grey circles) and of unaffected family members (white circles)(initial as well as follow-up values included). Horizontal bars represent medians, reference ranges for serum electrolytes are indicated by grey boxes. Next to significant hypomagnesemia, individuals with RRAGD mutations exhibited significantly lower serum potassium and serum chloride levels reflecting renal salt wasting (one-sided Student`s t-test (magnesium, chloride) and Mann Whitney U-test (potassium); ${ }^{* * *}$ $=p<0.001) .(F)$ Left ventriculography (performed at the age of 12 years) of individual F2.1 who presented with cardiomyopathy. Pictures show left ventricular volumes during diastole (left) and systole (middle) demonstrating a reduced ejection fraction of $40 \%$ (normal $55-70 \%$ ). Initial echocardiogram of individual F7.1 performed after genetic diagnosis (apical 4-chamber view) showing the dilated left ventricle.

\section{Figure 2. Localization of mutations in the RagD protein}

(A) Domain organization of RagD with GTP binding motifs in dark blue (G1-G5). Mutations are indicated with an arrow. (B) Crystal structure of RagD in complex with a GTP analogue (GppNHp, pdb entry 2q3f). Mutated residues are shown in red. GppNHp and the coordinated $\mathrm{Mg}^{2+}$ ion are depicted in dark blue. (C-D) Detailed view of the phosphate moiety of the nucleotide (C) and the nucleotide base (D). Affected residues are colored in light red. Dotted lines indicate hydrogen bonds. (E-G) Double immunofluorescence staining of mouse kidney 
(B-D) sections for RagD in green and uromodulin (B, red), NCC (C, red) or aquaporin ( $D$, red). Bars represent $20 \mu \mathrm{m}$.

\section{Figure 3. RRAGD mutations increase $\mathrm{mTOR}$ signaling in vitro}

(A) Immunoblot analysis demonstrating that RagD mutations (bold) increase Raptor and mTOR binding, indicated by arrows (top rows). RagA and RagD blots indicate equal (co-) precipitation of the Rag dimer (middle rows). Transfected HEK293T cells contained equal amounts of Raptor and mTOR as detected in the cell lysate fraction (bottom rows). (B) mTORC1 signaling in response to amino acids and starvation assessed by phosphorylation of its substrate S6 kinase (S6K1) at Threonine $389\left(\mathrm{Thr}^{389}\right)$, indicated by an arrow. Compared to wt RagD, all RagD mutants caused an increased S6K1 phosphorylation in the presence of amino acids. The expression of transfected RagA, RagD and S6K1, as well as of endogenous Raptor, was confirmed by immunoblotting (bottom rows). Constitutively active RagA-p.Q66L served as a positive control, (25) Metap-2 as a negative control for non-specific flag-binding. (C) Volcano plot of the interactome of murine RagD-p.S75L vs. RagD-wt. The significance (pvalue) of changes between RagD-p.S75L and RagD-wt (- $\log 10$ scale) is plotted against the difference (fold change) between RagD-p.S75L and RagD-wt (log2 scale). Dots beyond the curved line represent proteins with significantly increased abundance (FDR $0.1, \mathrm{~s} 0=0.5$ ) in RagD-p.S75L samples (right) or RagD-wt samples (left). (D) Model of the V-ATPaseRagulator-Rag-GTPase-mTORC1 complex and table listing the identified components from panel C that showed significantly increased binding to RagD-p.S75L in log2-fold change. 


\section{TABLES}

Table 1. Clinical and laboratory characteristics of patients with RRAGD mutations

\begin{tabular}{|c|c|c|c|c|c|c|c|c|c|c|}
\hline Individuals & F1.1 & F2.1 & F3.1 & F4.1 & F5.1 & F6.1 & F7.1 & F8.1 & F9.1 & $\begin{array}{l}\text { Family } 9 \\
\text { median }(n=8)\end{array}$ \\
\hline Origin & US & France & France & Belgium & Germany & Belgium & Germany & Turkey & Belgium & Belgium \\
\hline Gender & $M$ & $\mathrm{~F}$ & $\mathrm{~F}$ & $\mathrm{M}$ & $\mathrm{F}$ & $M$ & $\mathrm{~F}$ & $\mathrm{M}$ & $\mathrm{M}$ & $4 \mathrm{~F} / 4 \mathrm{M}$ \\
\hline Age at manifestation & 3 years & 3 years & 6 months & 5 years & 7 months & 14 years & 2 years & 6 years & 20 years & 2.5-24 years \\
\hline \multicolumn{11}{|l|}{ INITIAL FINDINGS } \\
\hline $\begin{array}{l}\text { Dilated Cardiomyopathy } \\
\text { (age at diagnosis) }\end{array}$ & $\begin{array}{l}\text { Yes } \\
(3 \mathrm{yrs})\end{array}$ & $\begin{array}{l}\text { Yes } \\
(12 \mathrm{yrs})\end{array}$ & $\begin{array}{l}\text { Yes } \\
(6 \mathrm{mo})\end{array}$ & No & $\begin{array}{l}\text { Yes } \\
(7 \mathrm{mo})\end{array}$ & $\begin{array}{l}\text { Yes } \\
\text { (14 years) }\end{array}$ & $\begin{array}{l}\text { Yes } \\
(4 \text { yrs) }\end{array}$ & No & No & No \\
\hline Fractional shortening $(\%, \mathrm{~N}:>25)$ & 7 & 8 & 15 & 31 & 15 & - & 18 & - & - & - \\
\hline Ejection fraction (\%, N: 55-70) & 15 & 31 & - & 61 & 16 & 50 & - & - & - & - \\
\hline $\begin{array}{l}\text { Left ventricular end-diastolic } \\
\text { diameter * }(\mathrm{mm})\end{array}$ & $\begin{array}{l}53 \\
(>P 99.9)\end{array}$ & $\begin{array}{l}55 \\
(>P 99.9)\end{array}$ & $\begin{array}{l}33 \\
\text { (P99.7) }\end{array}$ & $\begin{array}{l}34 \\
(\mathrm{P} 17.1)\end{array}$ & $\begin{array}{l}42 \\
(>P 99.9)\end{array}$ & & $\begin{array}{l}46 \\
(>P 99.9)\end{array}$ & - & - & - \\
\hline $\begin{array}{l}\text { Hypomagnesemia-related } \\
\text { symptoms }\end{array}$ & No & tetany & $\begin{array}{l}\text { cerebral } \\
\text { seizure }\end{array}$ & $\begin{array}{l}\text { tetany, } \\
\text { muscle } \\
\text { cramps }\end{array}$ & No & No & No & $\begin{array}{l}\text { cerebral } \\
\text { seizure }\end{array}$ & $\begin{array}{l}\text { tetany, } \\
\text { muscle } \\
\text { cramps }\end{array}$ & $7 / 8$ \\
\hline Nephrocalcinosis & Yes & Yes & Yes & No & Yes & No & Yes & Yes & No & $1 / 8$ \\
\hline Polyuria ** & No & No & Yes & No & Yes & No & Yes & Yes & Yes & $2 / 8$ \\
\hline Metabolic alkalosis & No & $?$ & Yes & Yes & No & No & No & Yes & Yes & $4 / 8$ \\
\hline \multicolumn{11}{|l|}{ Initial laboratory findings: } \\
\hline S-Na (mmol/L, N: 135-145) & 135 & - & 137 & 137 & 141 & 136 & 142 & 141 & 140 & 139 \\
\hline S-K (mmol/L, N: 3.5-5.0) & 4.7 & 3.2 & 3.5 & 3,4 & 3.5 & 3.5 & 3.9 & 2.7 & 3.5 & 3.3 \\
\hline S-Cl (mmol/L, N:98-107) & 98 & - & 95 & - & 100 & 95 & 105 & 91 & 95 & 98,5 \\
\hline S-Ca (mmol/L, N: 2.20-2.65) & 2.05 & 1.78 & 2.20 & 2.2 & 2.11 & 2.37 & 2.50 & 1.8 & 2.41 & 2.36 \\
\hline S-Mg (mmol/L, N: 0.7-1.1) & 0.50 & 0.27 & 0.40 & 0.43 & 0.40 & 0.42 & 0.54 & 0.26 & 0.54 & 0.49 \\
\hline $\mathrm{S}-\mathrm{PO}_{4}(\mathrm{mmol} / \mathrm{L}, \mathrm{N}: 0.6-1.4)$ & 1.56 & - & 1.44 & 1.59 & 1.50 & - & 1.53 & 1.7 & 0.87 & 1.00 \\
\hline S-creatinine (mg/dL) & 0.51 & 0.70 & 0.24 & 0.23 & 0.21 & 0.75 & 0.30 & 0.47 & 0.74 & 0.51 \\
\hline $\mathrm{S}-\mathrm{HCO}_{3}(\mathrm{mmol} / \mathrm{L}, \mathrm{N}: 22-26)$ & - & - & 24 & 29.3 & - & - & 28.5 & 29.1 & - & - \\
\hline iPTH (pg/mL, N: 10-65) & 33 & - & 17 & 29 & 37 & - & 91 & 108 & 21 & 18 \\
\hline
\end{tabular}




\begin{tabular}{|c|c|c|c|c|c|c|c|c|c|c|}
\hline FE-Na $(\%, \mathrm{~N}:<2)$ & 1.0 & - & 0.34 & 0,4 & 0.8 & - & 0.9 & 0.34 & 0.4 & 0.40 \\
\hline FE-K (\%, N: 4-16) & 13 & - & 27 & 6,3 & 24 & - & 23 & 14 & 7.5 & 6.2 \\
\hline $\mathrm{Ca} /$ Crea-ratio $(\mathrm{mol} / \mathrm{mol}, \mathrm{N}:<0.9)$ & 0.67 & 1.8 & $1.24^{\star * *}$ & 0.12 & $1.46^{\star \star \star}$ & - & 2.27 & 1.38 & 0.25 & - \\
\hline FE-Mg (\%, N: < 4) & 47 & 13.2 & 7.0 & 4.3 & 16 & - & 13.2 & 13.8 & 3.7 & - \\
\hline \multicolumn{11}{|l|}{ FOLLOW-UP FINDINGS } \\
\hline Age at last follow-up (yr) & 6yrs & & $20 y r s$ & 14 yrs & $10 y r s$ & 35yrs & $5 y r s$ & $19 y r s$ & $40 y r s$ & 2.5-61yrs \\
\hline Heart Transplant (Age) & Yes (3.3y) & Yes (25y) & Yes (15y) & No & No & No & No & No & No & No \\
\hline Fractional shortening $(\%, \mathrm{~N}:>25)$ & d.n.a. & d.n.a. & d.n.a. & 33 & 22 & 14 & 28 & 38 & & \\
\hline Ejection fraction $(\%, \mathrm{~N}: 55-70)$ & d.n.a. & d.n.a. & d.n.a. & 62 & 41 & 45 & 50 & 67 & 78 & $60-78$ \\
\hline $\begin{array}{l}\text { Left ventricular end-diastolic } \\
\text { diameter * }(\mathrm{mm})\end{array}$ & d.n.a & d.n.a. & d.n.a. & $\begin{array}{l}37 \\
(\mathrm{P} 1.8)\end{array}$ & $\begin{array}{l}42 \\
(P 77.3)\end{array}$ & 49 & $\begin{array}{l}43 \\
(>P 99.9)\end{array}$ & 48 & & \\
\hline \multicolumn{11}{|l|}{ Most recent laboratory findings: } \\
\hline S-Na (mmol/L, N: 135-145) & 138 & - & 140 & 140 & 139 & 140 & 142 & 141 & 140 & 140 \\
\hline $\mathrm{S}-\mathrm{K}(\mathrm{mmol} / \mathrm{L}, \mathrm{N}: 3.5-5.0)$ & 4.6 & - & 3.7 & 3,3 & 3.3 & 4.6 & 3.2 & 3.5 & 3.5 & 3.4 \\
\hline $\mathrm{S}-\mathrm{Cl}(\mathrm{mmol} / \mathrm{L}, \mathrm{N}: 98-107)$ & 104 & & 100 & & 100 & 103 & 104 & 95 & 97 & 98 \\
\hline $\mathrm{S}-\mathrm{Ca}(\mathrm{mmol} / \mathrm{L}, \mathrm{N}: 2.20-2.65)$ & 2.57 & - & 2.34 & 2,41 & 2.50 & 2.42 & 2.40 & 2.27 & 2.3 & 2.4 \\
\hline S-Mg (mmol/L, N: 0.7-1.1) & 0.58 & - & 0.43 & 0,55 & 0.50 & 0.62 & 0.66 & 0.53 & 0.46 & 0.48 \\
\hline S-creatinine (mg/dL) & 0.47 & - & 0.64 & 0,52 & 0.60 & 1.18 & 0.40 & 0.69 & 0.67 & 0.57 \\
\hline $\mathrm{S}-\mathrm{HCO}_{3}(\mathrm{mmol} / \mathrm{L}, \mathrm{N}: 22-26)$ & 23.0 & - & 28.0 & 25.0 & 27.0 & 24.1 & 24.2 & 29.9 & 35.0 & 29.4 \\
\hline iPTH (pg/mL, N: 10-65) & 94 & - & 54 & I & 34 & 17.4 & 60 & 41 & 18 & 17 \\
\hline FE-Na (\%, N: < 2) & 1.0 & - & 1.00 & 0.34 & 1.04 & 0.67 & 0.9 & 0.79 & 0.77 & 0.57 \\
\hline FE-K (\%, N: 4-16) & 15.5 & - & 11.0 & 33.7 & 18.9 & 13.8 & 34 & 18.2 & 12.1 & 10.7 \\
\hline FE-Cl $(\%, N:$ & 2.71 & - & - & & 1.52 & & & - & & \\
\hline Ca/Crea-ratio $(\mathrm{mol} / \mathrm{mol}, \mathrm{N}:<0.9)$ & 0.34 & - & 0.09 & 0.22 & 0.25 & 1.02 & 1.14 & 0.08 & 0.37 & - \\
\hline FE-Mg $(\%, \mathrm{~N}:<4)$ & 26.0 & - & 6.3 & 17.5 & 12.8 & 3.3 & 9.4 & 14.6 & 5.9 & 5.2 \\
\hline \multicolumn{11}{|l|}{ Therapy } \\
\hline Magnesium supplementation & Yes & $?$ & Yes & Yes & Yes & Yes & Yes & Yes & Yes & $6 / 8$ \\
\hline Potassium supplementation & No & $?$ & Yes & Yes & Yes & No & Yes & Yes & No & $3 / 8$ \\
\hline Heart failure medication & Yes & $?$ & Yes & No & Yes & Yes & Yes & No & No & $0 / 8$ \\
\hline
\end{tabular}




\begin{tabular}{|l|l|l|l|l|l|l|l|l|l|l|}
\hline Nucleotide level & c.227C>T & c.356C >G & c.662T>A & c.227C>T & c.356C>G & c.227C>T & c.356C >T & c.227C >G & c.289A $>C$ & c.289A>C \\
\hline Protein level & p.Ser76Leu & p.Pro119Arg & p.lle221Lys & p.Ser76Leu & p.Pro119Arg & p.Ser76Leu & p.Pro119Leu & p.Ser76Trp & p.Thr97Pro & p.Thr97Pro \\
\hline Inheritance & de novo & de novo & de novo & dominant? & de novo & de novo & $\begin{array}{l}\text { maternal } \\
\text { mosaicism }\end{array}$ & de novo & dominant & dominant \\
\hline
\end{tabular}

* Calculations according to Pettersen et al. (45)

** $>2 \mathrm{~L} / \mathrm{m}^{2} \mathrm{BSA} /$ day (BSA: body surface area)

*** molar Ca/crea ratio $\mathrm{N}:<2.2$ for infants

d.n.a. : does not apply 
Figure 1 bioRxiv preprint doi: https://doi.org/10.1101/2021.03.11.434334; this version posted March 12, 2021. The copyright holder for this preprint (which was not certified by peer review) is the author/funder, who has granted bioRxiv a license to display the preprint in perpetuity. It is

A

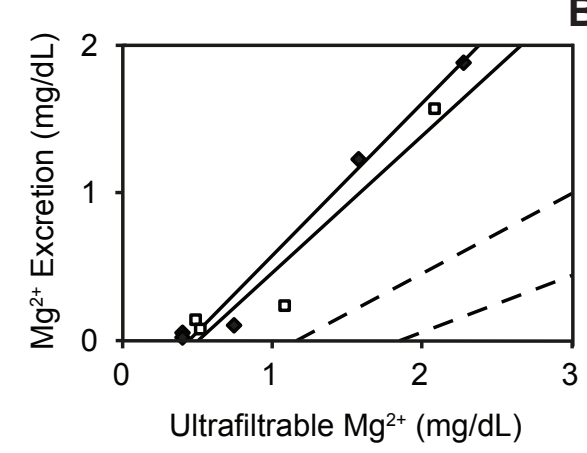

C

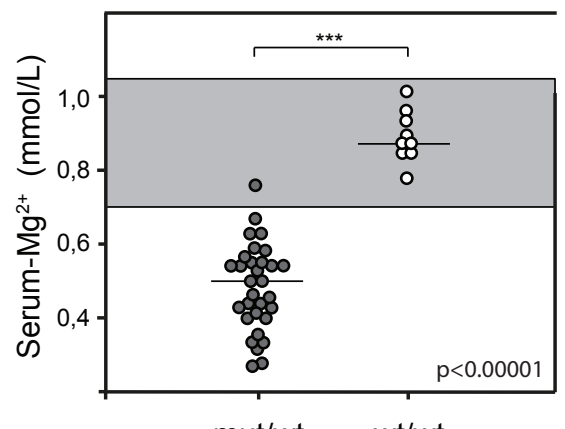

mut/wt

$\mathbf{F}$

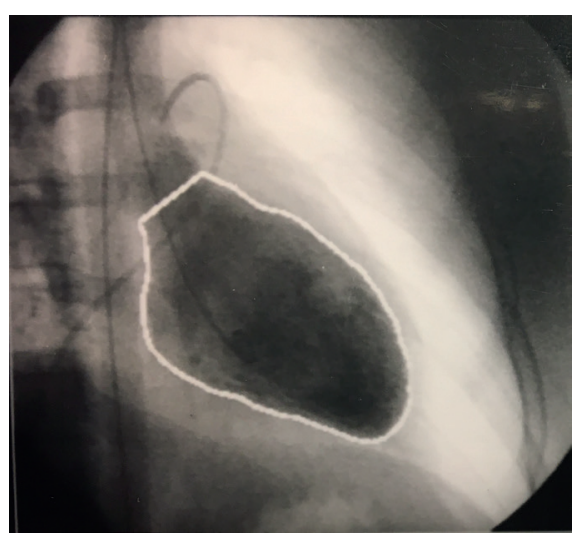
available under aCC-BY-NC-ND 4.0 International license.
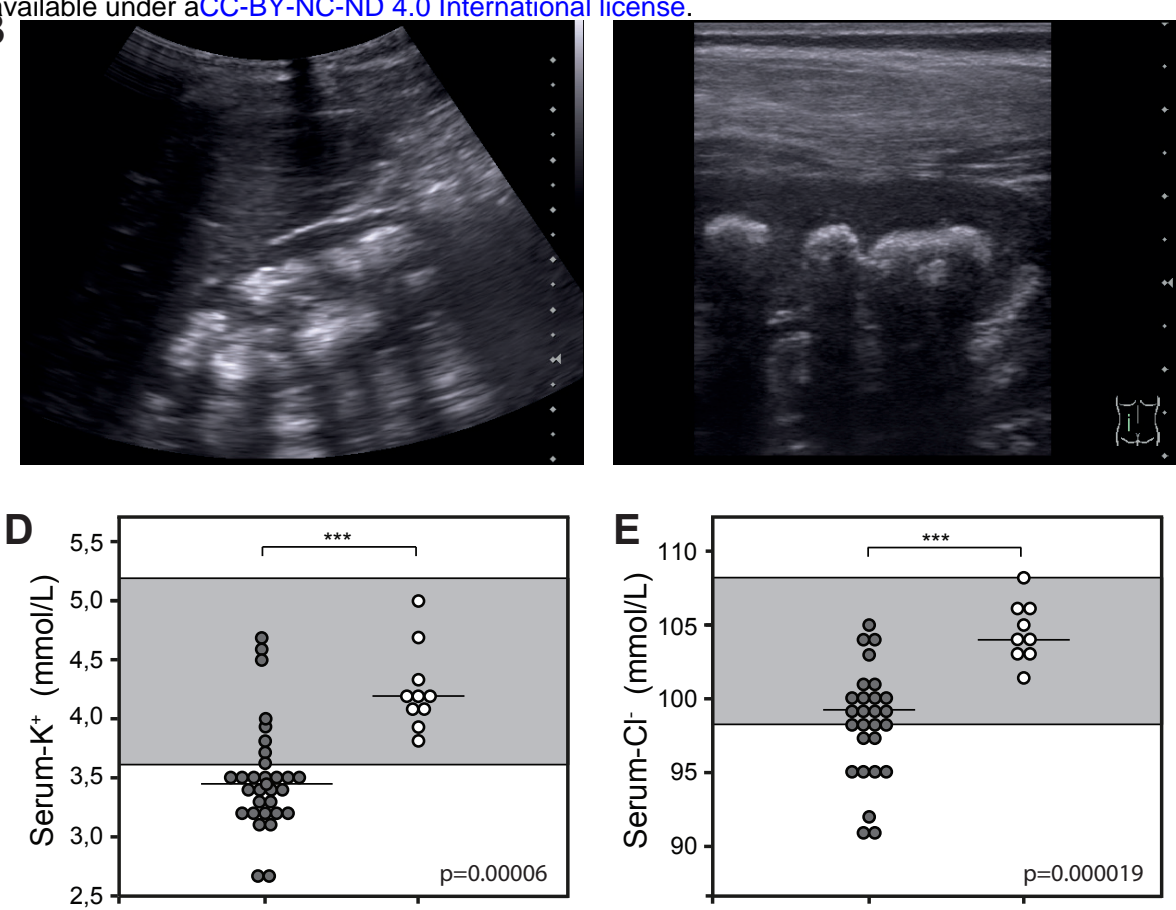

mut/wt wt/wt

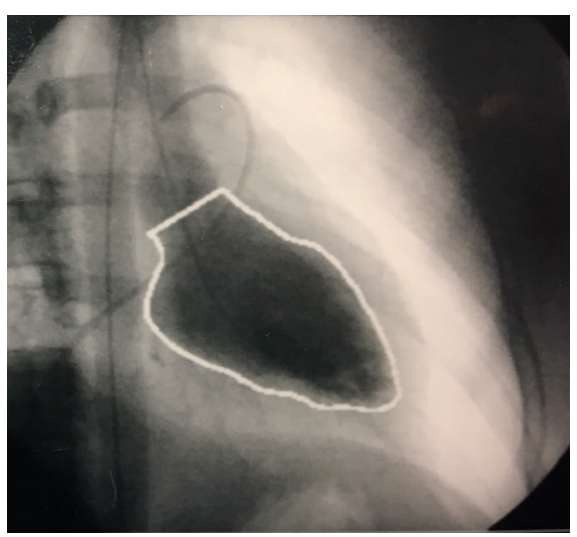

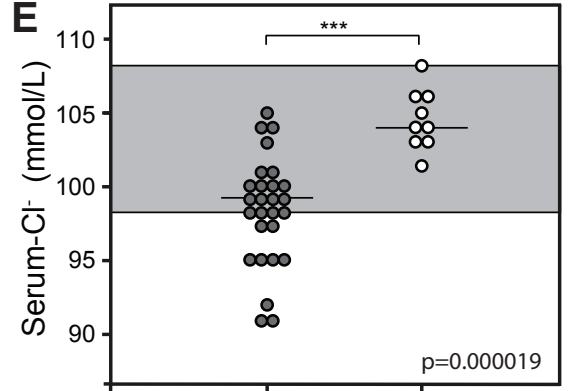

mut/wt $\quad$ wt/wt

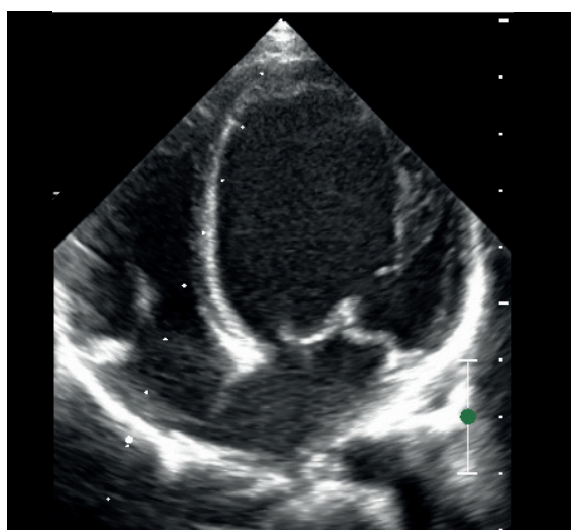


Figure 2 bioRxiv preprint doi: https://doi.org/10.1101/2021.03.11.434334; this version posted March 12, 2021. The copyright holder for this preprint (which was not certified by peer review) is the author/funder, who has granted bioRxiv a license to display the preprint in perpetuity. It is made available under aCC-BY-NC-ND 4.0 International license.

A
G1 G2

G3

G4

G5

C-terminal domain

RagD

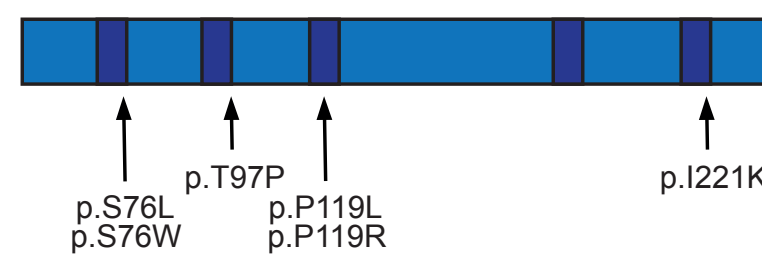

B

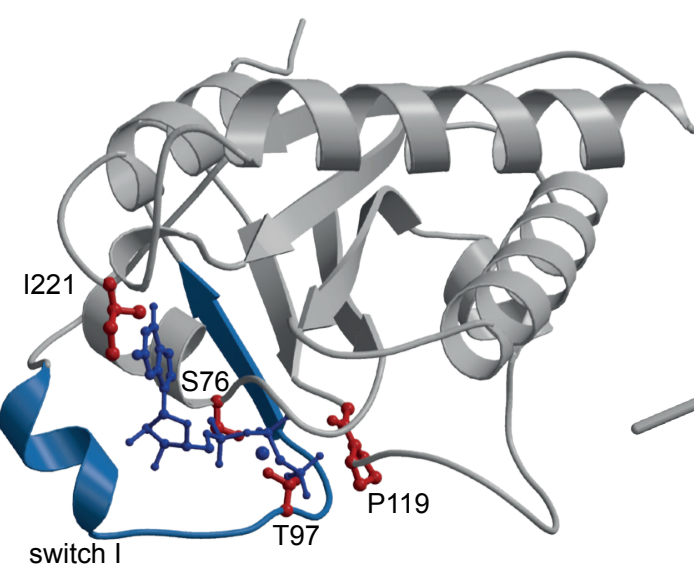

C

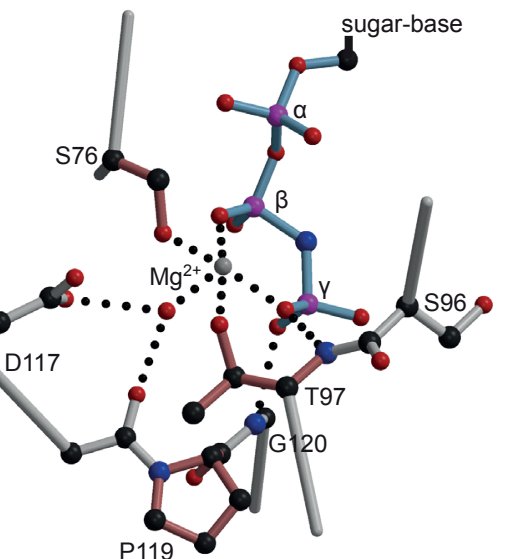

D

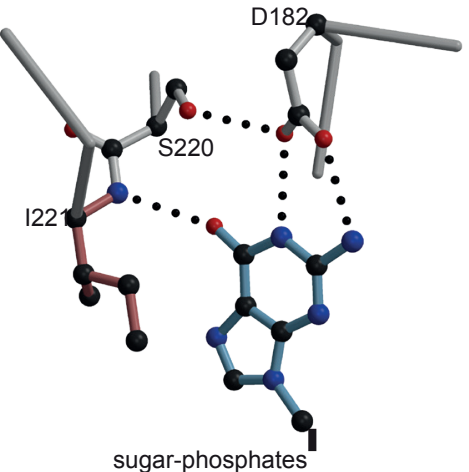

E
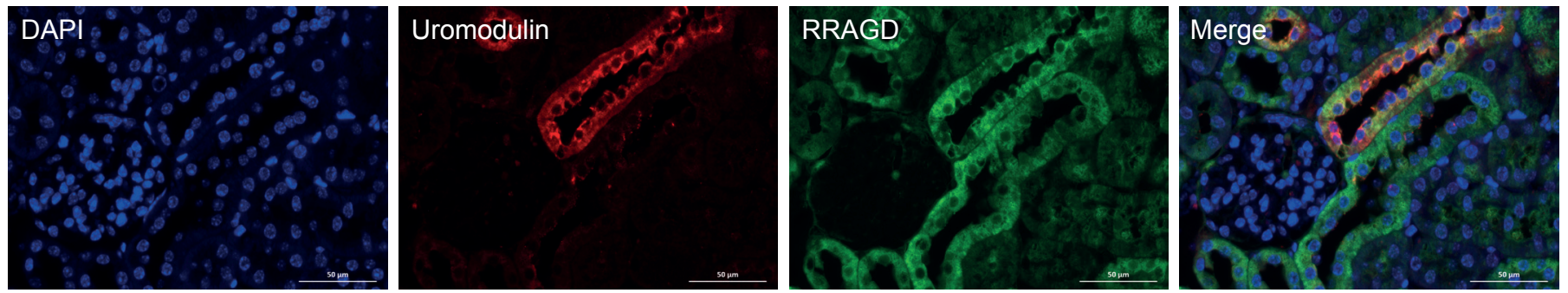

F
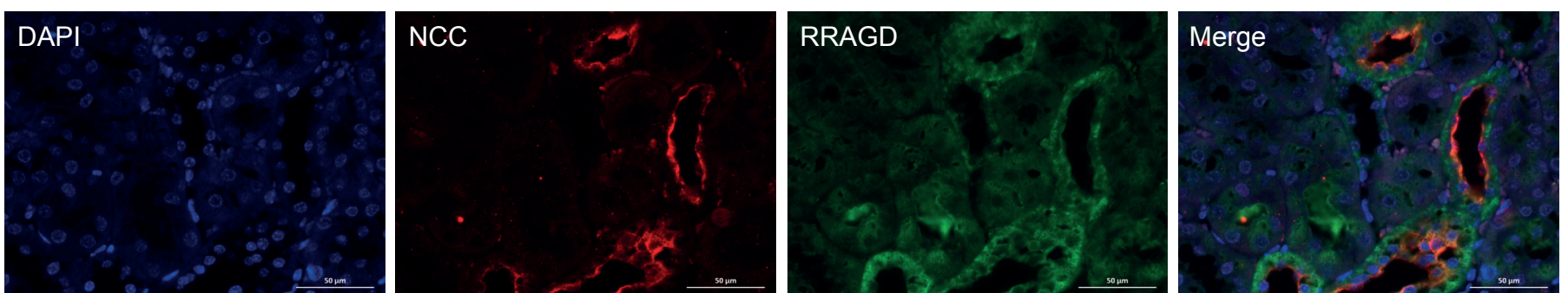

G
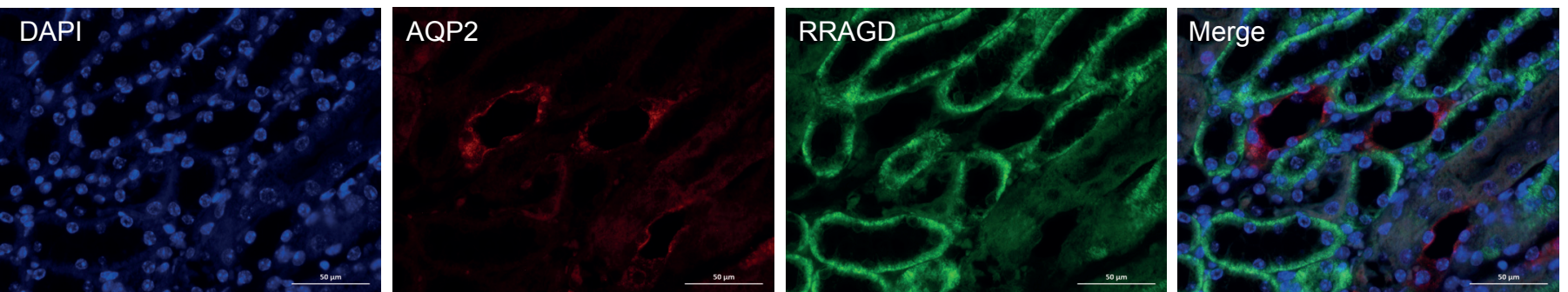
Figure 3 bioRxiv preprint doi: https://doi.org/10.1101/2021.03.11.434334; this version posted March 12, 2021. The copyright holder for this preprint (which was not certified by peer review) is the author/funder, who has granted bioRxiv a license to display the preprint in perpetuity. It is made available under aCC-BY-NC-ND 4.0 International license.

A

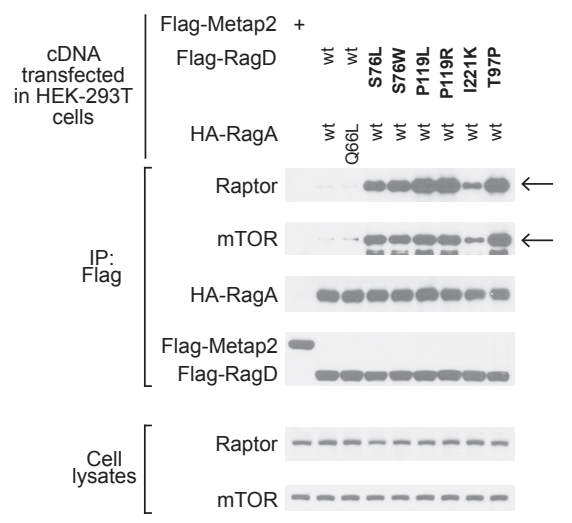

C

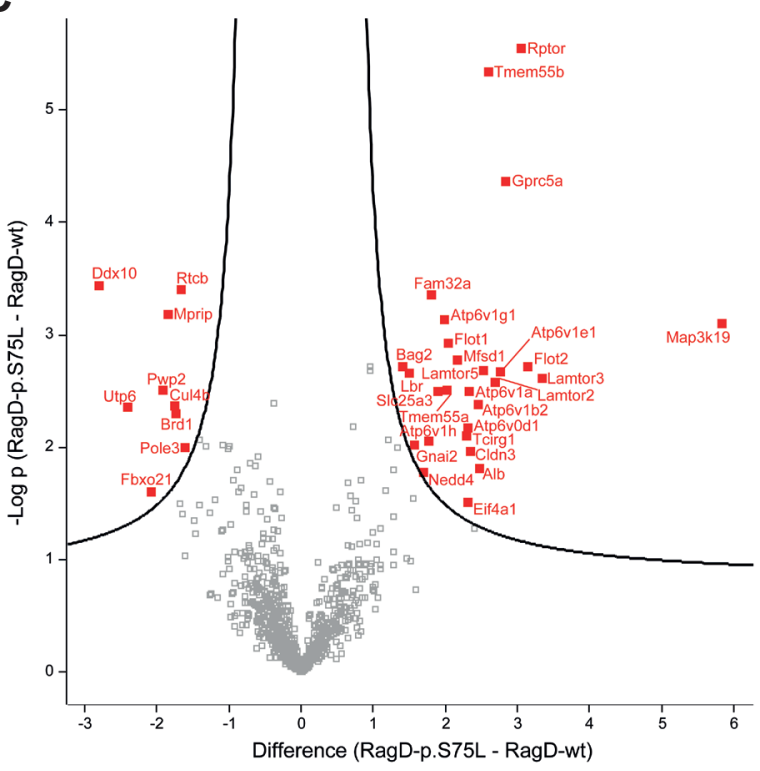

B

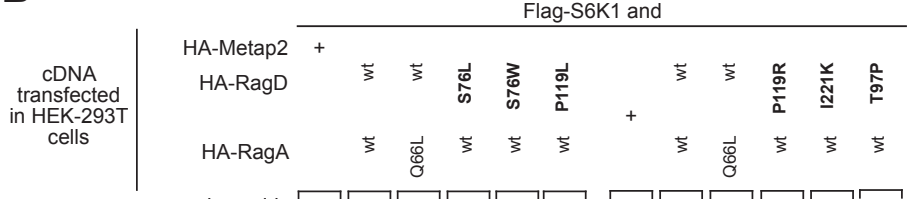

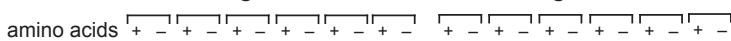

IP:
Flag

$\mathrm{pT}^{389}-\mathrm{S} 6 \mathrm{~K} 1$ -

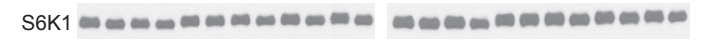

HA-Metap2

HA-RagD

HA-RagA

Raptor
D

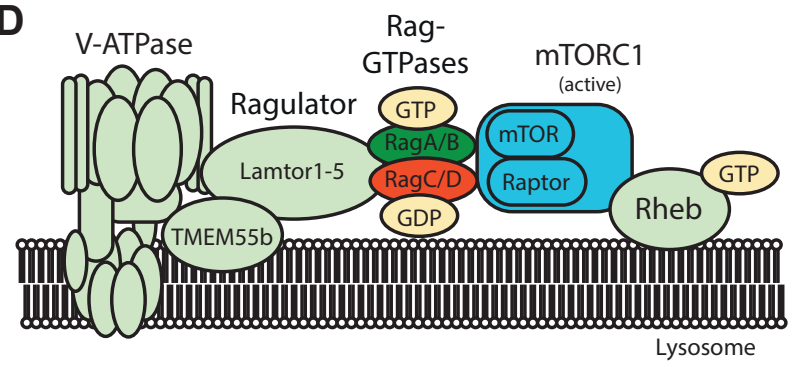

\begin{tabular}{lll}
\multicolumn{2}{c}{ Specific Interactors of mRagD-p.S75L vs. mRagD-wt } \\
Protein & Gene & Difference \\
& & \\
Ragulator complex protein LAMTOR3 & Lamtor3 & 3.3500 \\
Regulatory-associated protein of mTOR & Rptor & 3.0583 \\
V-type proton ATPase subunit E1 & Atp6v1e1 & 2.7697 \\
Ragulator complex protein LAMTOR2 & Lamtor2 & 2.6929 \\
Type1 PIP -4-phosphatase $_{\text {Ragulator complex protein LAMTOR5 }}^{\text {Tmem55b }}$ & 2.5940 \\
V-type proton ATPase subunit B2 & Lamtor5 & 2.5223 \\
V-type proton ATPase catalytic subunit A & Atp6v1b2 & 2.4520 \\
V-type proton ATPase subunit d1 & Atp6v1a & 2.3375 \\
V-type proton ATPase subunit a & Atp6v0d1 & 2.3149 \\
Type2 PIP ${ }_{2}^{-4-p h o s p h a t a s e ~}$ & Tcirg1 & 2.3028 \\
V-type proton ATPase subunit G1 & Tmem55a & 2.0265 \\
V-type proton ATPase subunit H & Atp6v1g1 & 1.9777 \\
& Atp6v1h & 1.7714
\end{tabular}

